\title{
Prospective comparative evaluation of the European Society of Cardiology (ESC) 1-hour and a 2-hour rapid diagnostic algorithm for myocardial infarction using high-sensitivity troponin-T
}

James E. Andruchow, MD, MSc ${ }^{*}$; Timothy Boyne, MD, MSc ${ }^{\ddagger}$; Isolde Seiden-Long, PhD ; Dongmei Wang, MSc\%; Shabnam Vatanpour, PhD*; Grant Innes, MD, MSc ${ }^{*}$; Andrew D. McRae, $\mathrm{PhD}, \mathrm{MD}^{*}+$

\section{CLINICIAN'S CAPSULE}

What is known about this topic?

Several high-sensitivity troponin (hs-cTn) algorithms to rule out myocardial infarction (MI) exist, leaving physicians unsure which to implement.

What did this study ask?

This study prospectively compares the diagnostic performance of 1- and 2-hour hs-cTn algorithms for Canadian emergency department patients with chest pain.

What did this study find?

Both algorithms were accurate at diagnosing and excluding $\mathrm{MI}$, but the 2-hour algorithm may offer several practical advantages.

Why does this study matter to clinicians?

Institutions implementing hs-cTn assays can choose between 1-or 2-hour algorithms, which can safely expedite patient care.

\section{ABSTRACT}

Objective: Both 1- and 2-hour rapid diagnostic algorithms using high-sensitivity troponin (hs-cTn) have been validated to diagnose acute myocardial infarction (MI), leaving physicians uncertain which algorithm is preferable. The objective of this study was to prospectively evaluate the diagnostic performance of 1- and 2-hour algorithms in clinical practice in a Canadian emergency department (ED).

Methods: ED patients with chest pain had high-sensitivity cardiac troponin-T (hs-cTnT) collected on presentation and 1- and 2-hours later at a single academic centre over a 2-year period. The primary outcome was index $\mathrm{MI}$, and the secondary outcome was 30-day major adverse cardiac events (MACE). All outcomes were adjudicated.

Results: We enrolled 608 patients undergoing serial hs-cTnT sampling. Of these, 350 had a valid 1-hour and 550 had a 2-hour hs-cTnT sample. Index MI and 30-day MACE prevalence was $\sim 12 \%$ and $14 \%$. Sensitivity of the 1- and 2-hour algorithms was similar for index $\mathrm{MI}$ 97.3\% (95\% Cl: 85.8-99.9\%) and 100\% (95\% Cl: 91.6-100\%) and 30-day MACE: $80.9 \%$ (95\% Cl: $66.7-$ $90.9 \%)$ and $83.3 \%(95 \% \mathrm{Cl}: 73.2-90.8 \%)$, respectively. Both algorithms accurately identified about $10 \%$ of patients as high risk.

Conclusions: Both algorithms were able to classify almost twothirds of patients as low risk, effectively ruling out $\mathrm{MI}$ and conferring a low risk of 30-day MACE for this group, while reliably identifying high-risk patients. While both algorithms had equivalent diagnostic performance, the 2-hour algorithm offers several practical advantages, which may make it preferable to implement. Broad implementation of similar algorithms across Canada can expedite patient disposition and lead to resource savings.

\section{RÉSUMÉ}

Objectif: Les algorithmes de diagnostic rapide au bout de $1 \mathrm{~h}$ et au bout de $2 \mathrm{~h}$ à l'aide de la troponine T cardiaque hypersensible (TnTc HS) ont tous les deux été validés dans la pose du diagnostic d'infarctus du myocarde (IM) aigu, toutefois les médecins ignorent lequel est préférable. L'étude visait donc à évaluer de manière prospective la performance diagnostique des algorithmes de diagnostic au bout de $1 \mathrm{~h}$ et de $2 \mathrm{~h}$ en pratique clinique dans les services des urgences (SU) au Canada. Méthode: Des analyses de la troponine T cardiaque hypersensible ont été effectuées chez les patients traités au SU pour des

From the * Department of Emergency Medicine; ${ }^{\dagger}$ Department of Community Health Sciences; ${ }^{\ddagger}$ Department of Cardiac Sciences, University of Calgary, Calgary, $A B ;{ }^{\S}$ Alberta Precision Laboratories, Calgary, $A B$; and the "Alberta Health Services, Calgary, $A B$.

Correspondence to: Dr. James Andruchow, Foothills Medical Centre, Room C-231, Department of Emergency Medicine, 1403-29 St. NW, Calgary, AB T2N 2T9; Email: jeandruc@ucalgary.ca. 
douleurs thoraciques, à l'arrivée ainsi qu'au bout de $1 \mathrm{~h}$ et de 2 $h$, dans un seul centre hospitalier universitaire, sur une période de 2 ans. Le principal critère d'évaluation était l'IM de référence, et le critère d'évaluation secondaire, les événements cardiaques graves (ECG) au bout de 30 jours. Tous les résultats ont été corroborés.

Résultats: Étaient admissibles à l'étude 608 patients soumis à un dosage de la TnTc HS en série. Dans l'ensemble, les résultats au bout de $1 \mathrm{~h}$ ont été validés chez 350 malades, et les résultats au bout de $2 \mathrm{~h}$, chez 550 malades. La prévalence de I'IM de référence et des ECG au bout de 30 jours était de $\sim 12 \%$ et de $14 \%$. La sensibilité des algorithmes de diagnostic rapide tant au bout de $1 \mathrm{~h}$ que de $2 \mathrm{~h}$ était comparable pour I'IM de référence, soit de 97,3\% (IC à 95\% : 85,8-99,9\%) et de $100 \%$ (IC à 95\% : 91,6-100\%), ainsi que pour les ECG au bout de 30 jours, soit de $80,9 \%$ (IC à $95 \%$ : 66,7-90,9\%) et de $83,3 \%$ (IC à 95\% : 73,2-90,8\%), respectivement. L'une et l'autre des démarches ont permis de différencier avec exactitude environ $10 \%$ des patients jugés à risque élevé.

Conclusion: Les deux algorithmes se sont révélés des moyens non seulement de ranger presque les deux tiers des patients dans la catégorie à faible risque, ce qui signifie que la possibilité d'IM était écartée de fait et que le risque d'ECG au bout de 30 jours était faible, mais aussi de repérer de manière fiable les patients à risque élevé. À performance diagnostique comparable, la démarche de $2 \mathrm{~h}$ offre plusieurs avantages pratiques, d'où l'intérêt de la privilégier. L'application courante d'algorithmes de diagnostic comparables, fondés sur la TnTc HS, partout dans les SU, au Canada, pourrait se traduire par des économies importantes sur le plan des ressources.

Keywords: Acute coronary syndrome, high-sensitivity troponin, major adverse cardiac events, myocardial infarction, rapid diagnostic algorithms

\section{INTRODUCTION}

Chest pain and symptoms of suspected cardiac ischemia lead to millions of emergency department (ED) visits annually worldwide. ${ }^{1}$ Research has demonstrated that very low concentrations of high-sensitivity cardiac troponin (hs-cTn) sampled on ED arrival, especially in combination with a non-ischemic electrocardiogram (ECG), are highly sensitive for index myocardial infarction (MI). ${ }^{2,3}$ However, guidelines recommend a single hs-cTn testing strategy only for patients with at least 3 -hours since symptom onset given the risk of falsenegative results in early presenters. ${ }^{4}$ Because the majority of patients will not meet these stringent criteria, serial hs-cTn sampling is recommended for most patients. Several rapid diagnostic algorithms measuring small but clinically significant changes in hs-cTn over fixed time intervals (usually 1 or 2 hours) have been validated, and while they are highly sensitive for index MI, they are less sensitive for 30-day major adverse cardiac events (MACE). ${ }^{5-19}$

While European Society of Cardiology (ESC) 2015 guidelines endorse a 1-hour hs-cTn algorithm, ${ }^{4}$ concerns about the optimal resampling interval and what interval change in hs-cTn concentrations is clinically meaningful persist. ${ }^{20}$ Moreover, few studies have directly compared the performance of these algorithms to each other within the same patient cohort. A recent publication examined the diagnostic performance of 14 rule-out MI algorithms, ${ }^{18}$ including the ESC 1-hour and Reichlin 2-hour ${ }^{8}$ high-sensitivity cardiac troponin-T (hs-cTnT) algorithms, but did not compare their rule-in performance. Finally, the bulk of research to date has been performed in Europe and Australasia with samples processed in a single core laboratory likely representing optimal test conditions and may not be reflective of realworld assay performance. Consequently, with several rapid diagnostic algorithms to choose from, selecting the optimal algorithm balancing ED length of stay, patient safety, and logistical considerations has become a challenge for many Canadian EDs implementing hs-c Tn assays.

The objective of this study is to prospectively validate and compare the ESC-endorsed 1-hour rapid diagnostic algorithm using hs-c $\mathrm{TnT}^{4}$ with a 2-hour hs-cTnT algorithm ${ }^{8}$ (Figure 1) under real world conditions by quantifying their diagnostic performance for index MI and 30-day MACE (sensitivity, specificity, negative and positive predictive values [NPV/PPV]), and negative and positive likelihood ratios [(LR +/LR-]). Our hypothesis is that both the 1- and 2-hour algorithms will have similar diagnostic accuracy for both index MI and 30-day MACE.

\section{METHODS}

\section{Study design, time period, and setting}

This prospective observational cohort study was conducted at a large urban level one trauma and regional 


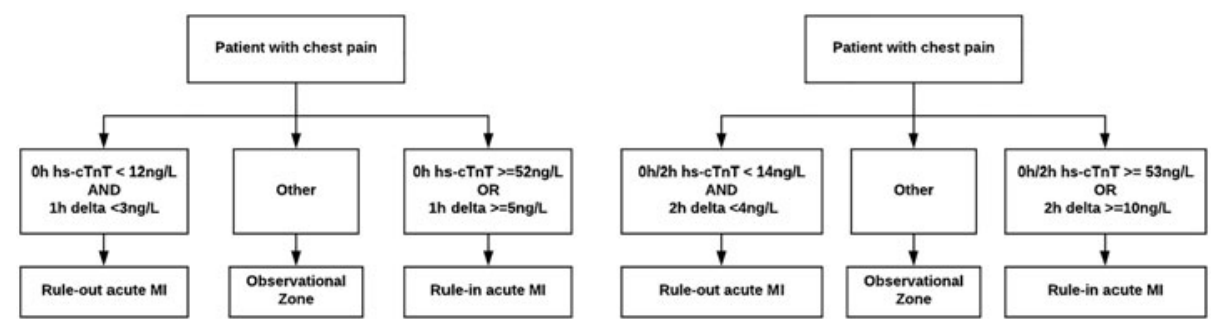

Figure 1. One and 2-hour rapid diagnostic algorithms using hs-cTnT.

percutaneous coronary intervention (PCI) centre in Calgary, Alberta, from August 2014 to September 2016. The ED has an annual patient volume of approximately 80,000 visits, including approximately 2,500 annual visits for chest pain, and is staffed exclusively by certified emergency physicians. This study was conducted according to the Standards for Reporting Diagnostic accuracy studies (STARD) guidelines for studies of diagnostic accuracy (Supplement) and was approved by the University of Calgary Conjoint Health Research Ethics Board.

\section{Population}

Patients were eligible if they were ages 25 years and older, presented to the ED with Canadian Emergency Department Information System (CEDIS) standardized chief complaints ${ }^{21}$ of "chest pain - cardiac features" or "cardiac type pain" and required serial troponin testing to rule out MI at the discretion of the attending emergency physician. Patients were excluded from the study if, according to the attending emergency physician, they had ST-elevation MI, clear acute ischemic changes, or new arrhythmia (not including sinus tachycardia, premature atrial contractions, premature ventricular contractions, paced rhythm, or rate-controlled atrial fibrillation/atrial flutter) on the initial ECG, were diagnosed with an acute coronary syndrome in the 30 days prior to the index visit, were hemodynamically unstable, had advanced renal failure requiring dialysis, or were unable to provide consent secondary to language barriers or cognitive issues. Patients unable to have valid samples collected within the +/- 30-minute window of the specified collection time were excluded from the analysis.

\section{Troponin assay}

Hs-c'TnT (Roche Elecsys ${ }^{\circledR}$ High-sensitivity, 5th generation, Troponin $\mathrm{T}$ assay performed on the cobas e 601 instrument as per the manufacturer's specifications) results were obtained for all patients. This assay has a limit of blank (LoB) of $3 \mathrm{ng} / \mathrm{L}$, a limit of detection (LoD) of $5 \mathrm{ng} / \mathrm{L}$, a 99th percentile of $14 \mathrm{ng} / \mathrm{L}$ in a healthy population, and an imprecision corresponding to a $10 \%$ coefficient of variation at the limit of quantitation (LoQ) of $13 \mathrm{ng} / \mathrm{L}$.

\section{Study procedures}

Trained research assistants approached consecutive patients between 0800 and 2000 hours, 7 days a week, to obtain written informed consent and collect demographic data. Attending ED physicians used standardized case report forms to collate detailed clinical information regarding patient presentation and past medical history. All patients consented for a 30-day telephone follow-up and detailed review of medical records. Presenting (0-hour) hs-cTnT samples were collected as part of routine care by an emergency physician order or as part of a nurse-initiated chest pain protocol; care providers were not blinded to these results. After enrolment, 1- and 2-hour research hs-c TnT samples were collected by either a trained phlebotomist or registered nurse; these results were not disclosed to care providers. If an emergency physician wished to obtain 1- or 2-hour hs-cTnT results for a study patient, a separate physician order was required.

All patients underwent a detailed review of medical records incorporating the 30-day period following the index visit. Outcome data were also obtained using hospital administrative databases, Alberta vital statistics, and the APPROACH registry. APPROACH is a registry that prospectively collects data on all patients admitted with a cardiac diagnosis or who have a revascularization procedure in the province of Alberta. ${ }^{22}$

\section{Outcomes}

The primary outcome was index MI diagnosed on the basis of a rise and/or fall of hs-cTnT above the 99th 
percentile in the appropriate clinical context, in accordance with the Third Universal Definition of Myocardial Infarction. ${ }^{23}$ The secondary outcome was 30-day MACE (including MI, revascularization, or cardiac death) and its individual components. Cardiac death was adjudicated in accordance with the American College of Cardiology/ American Heart Association 2014 Definitions for Cardiovascular Endpoints. ${ }^{24}$ All outcomes were independently adjudicated by two physicians (board-certified cardiologist and board-certified emergency physician) after the review of all available clinical information, including ECGs, troponin results, imaging findings, and clinical documentation. All disagreements were resolved by consensus.

\section{Data analysis and sample size}

Descriptive statistics were performed for the cohort. Sensitivity, specificity, NPV, PPV, and LR+/LR- with 95\% confidence intervals were calculated for the 1and 2-hour algorithms. A pre-specified sensitivity analysis was conducted to examine the impact of excluding patients with ischemic ECG findings on outcome prevalence. Statistical analyses were performed using R Version 3.2.3 (www.r-project.org). To obtain a 95\% confidence interval of $+/-1.0 \%$ for the outcome of 30 -day MI (estimated prevalence 2\%), a sample size of 753 patients was calculated. The two-proportion z-test was used to compare test characteristics between algorithms.

\section{RESULTS}

A total of 1,167 eligible patients with at least one hs-cTnT sample collected were enrolled as part of a related study examining hs-cTnT concentrations on presentation (0-hour), which has been published separately. ${ }^{3}$ Of these, 559 patients were excluded because they did not require serial troponin sampling to rule out MI in the opinion of the attending emergency physician (usually because of prolonged and/or atypical symptoms), leaving 608 patients eligible for this study. The final data set included 350 patients with valid 1-hour and 550 patients with valid 2 -hour hs-cTn T samples (Figure 2). Samples for the 1-hour cohort and 2-hour cohort were collected on average 7.4 minutes (SD 7.3 minutes) and 6.8 minutes (SD 7.1 minutes) from the specified collection time, respectively.
Only 46 (13.1\%) of 1-hour and 66 (12.0\%) of 2-hour samples were collected more than 15 minutes from the designated collection time. Patient baseline characteristics and 30-day outcomes were similar among the two cohorts (Table 1). No patients were lost to follow-up.

The 1- and 2-hour algorithms categorized similar proportions of patients as low risk, $62.6 \%$ v. $63.8 \%$, respectively (Table 2). However, whereas the low-risk criteria of 2-hour algorithm were 100\% sensitive (95\% CI: 91.6-100\%) capturing all 48 MI patients, the 1-hour algorithm missed 1 of 37 patients with MI on the index visit (sensitivity 97.3\%, 95\% CI: 85.899.9\%). Sensitivity for 30-day MACE was lower for both algorithms, with 9 of 47 patients with MACE missed by the 1-hour algorithm (sensitivity $80.9 \%$, 95\% CI: $66.7-90.9 \%$ ) and 13 of 78 patients with MACE missed by the 2-hour algorithm (sensitivity 83.3\%, 95\% CI: 73.2-90.8\%). Both the 1- and 2-hour algorithms missed one patient with 30-day MI. One patient with 30-day cardiac death was missed by the 2-hour algorithm, whereas no cardiac deaths were missed by the 1-hour algorithm. None of these differences were statistically significant (two-proportion z-test $>0.05$ in all cases).

Both the 1-hour and 2-hour hs-cTnT algorithms categorized similar proportions of patients as high risk $(10.6 \%$ v. $10.5 \%)$ and were highly specific for index MI and 30-day MACE (Table 3). While the PPV point estimate for the 2-hour algorithm for index MI was higher ( $82.8 \%$ v. $70.3 \%)$, this difference was not statistically significant (two-proportion $\mathrm{z}$-test, $z=1.43, p=$ 0.1527). Both algorithms classified about one-quarter of patients in a non-diagnostic observational zone with an $\sim 11 \%$ index MI and $~ 12 \% 30$-day MACE prevalence (see Figure 1).

\section{DISCUSSION}

\section{Interpretation of findings}

Both algorithms were highly accurate for both ruling-in and ruling-out MI. The 1-hour algorithm had a sensitivity of $97.3 \%$ and -LR of 0.04 for both index and 30-day MI. The 2-hour algorithm had 100\% sensitivity and -LR of 0.00 for index MI and $98.4 \%$ sensitivity and -LR of 0.04 for 30-day MI. These findings suggest that the low-risk criteria of both algorithms confidently 


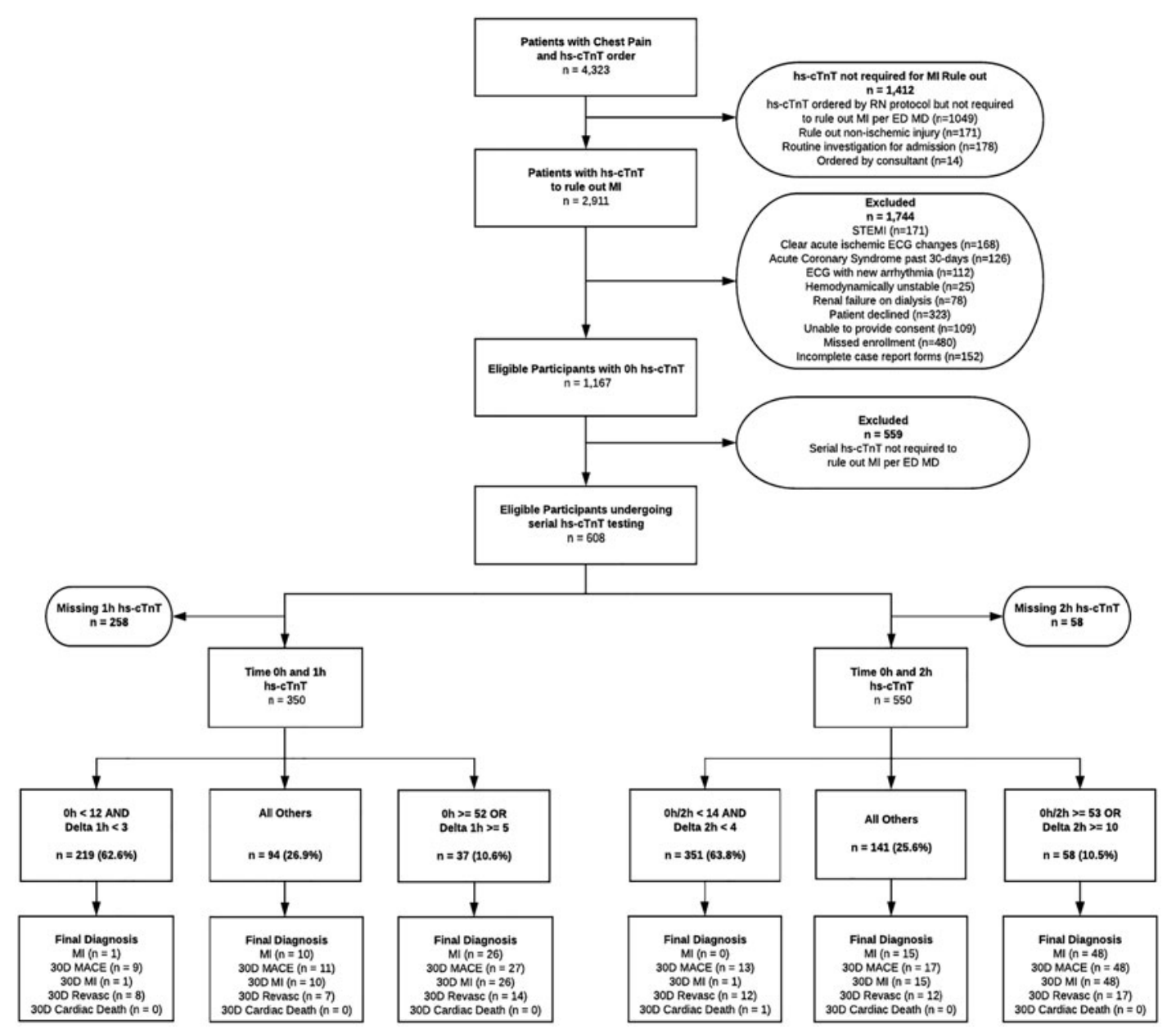

Figure 2. Standards for Reporting Diagnostic accuracy studies (STARD) diagram.

$\mathrm{ACS}=$ acute coronary syndrome; $\mathrm{MI}=$ myocardial infarction; 30D MACE $=30$-day major adverse cardiac event; 30D Revasc $=30$-day coronary revascularization.

rule out MI. Similarly, the high-risk criteria of both algorithms were highly specific for index MI and 30-day MACE, with + LRs ranging from 17.4 to 37.1. These findings suggest that false-positive diagnoses of MI using either algorithm are unlikely. Patients with high-risk hs-cTnT findings should receive immediate treatment and cardiology consultation in the appropriate clinical context.

Not surprisingly, both algorithms were less sensitive for 30-day MACE, emphasizing the continued importance of ECG findings and thorough clinical assessment, in addition to biomarkers in identifying patients at risk of short-term MACE. However, given NPVs for 30-day MACE of $~ 96 \%$ for both algorithms, in the absence of high-risk clinical features, discharge with outpatient follow-up appears safe for the majority of patients with low-risk hs-cTnT results.

\section{Comparison to previous studies}

To our knowledge, this is only the second direct comparison of the ESC-recommended 1-hour rapid diagnostic algorithm with a 2-hour hs-cTnT algorithm in the same cohort. ${ }^{18}$ These results validate prior work $^{5,7,16,18}$ and confirm that both algorithms can rapidly rule out $\mathrm{MI}$ and facilitate early discharge by identifying almost two-thirds of patients as low risk for 30-day MACE. The widespread adoption of similar algorithms across Canadian EDs could reduce ED length of stay, defer testing of low-risk patients to community settings, and decongest EDs and inpatient units, a finding which has already been demonstrated in Europe. ${ }^{25}$

While no statistically significant differences in diagnostic test characteristics for any outcomes were observed between the algorithms in our study, the 


\begin{tabular}{|c|c|c|}
\hline Patient characteristics & $\begin{array}{l}\text { 1-Hour } \\
\text { cohort }\end{array}$ & $\begin{array}{l}\text { 2-Hour } \\
\text { cohort }\end{array}$ \\
\hline $\mathrm{N}$ & 350 & 550 \\
\hline Median age (IQR) & $60(52-72)$ & $61(52-74)$ \\
\hline Male & $225(64.3 \%)$ & $341(62.0 \%)$ \\
\hline Arrival by ambulance & $147(42.0 \%)$ & $243(44.2 \%)$ \\
\hline CAD history & $114(32.6 \%)$ & $190(34.5 \%)$ \\
\hline Vascular disease history & $15(4.3 \%)$ & $29(5.3 \%)$ \\
\hline Hypertension & $211(60.3 \%)$ & $328(59.6 \%)$ \\
\hline Hyperlipidemia & $161(46.0 \%)$ & $262(47.6 \%)$ \\
\hline Diabetes & $65(18.6 \%)$ & $104(18.9 \%)$ \\
\hline Obesity & $77(22.0 \%)$ & $126(22.9 \%)$ \\
\hline Family history of CAD & $74(21.1 \%)$ & $115(20.9 \%)$ \\
\hline Smoker & $54(15.4 \%)$ & $77(14.0 \%)$ \\
\hline Chest pain onset $<3 \mathrm{hrs}$ & $142(40.6 \%)$ & $224(40.7 \%)$ \\
\hline \multicolumn{3}{|l|}{ Patient outcomes } \\
\hline Hospital admission on index visit & $90(25.7 \%)$ & $145(26.4 \%)$ \\
\hline 30-Day ED revisit & $44(12.6 \%)$ & $71(12.9 \%)$ \\
\hline 30-Day hospital admission & $13(3.7 \%)$ & $24(4.4 \%)$ \\
\hline 30-Day MI & $37(10.6 \%)$ & $64(11.6 \%)$ \\
\hline MI during index presentation & $37(10.6 \%)$ & $63(11.5 \%)$ \\
\hline Type 1 & $29(8.3 \%)$ & $48(8.7 \%)$ \\
\hline Type 2 & $8(2.3 \%)$ & $15(2.7 \%)$ \\
\hline $\begin{array}{l}\text { MI after index visit but within } 30 \\
\text { days }\end{array}$ & $0(0.0 \%)$ & $1(0.2 \%)$ \\
\hline 30-Day MACE & $47(13.4 \%)$ & $78(14.2 \%)$ \\
\hline MACE on index visit & $45(12.9 \%)$ & $75(13.6 \%)$ \\
\hline $\begin{array}{l}\text { MACE after index visit but within } \\
30 \text { days }\end{array}$ & $2(0.6 \%)$ & $3(0.5 \%)$ \\
\hline 30-Day revascularization & $29(8.3 \%)$ & $41(7.5 \%)$ \\
\hline Revascularization on index visit & $26(7.4 \%)$ & $37(6.7 \%)$ \\
\hline $\mathrm{PCl}$ & $22(6.3 \%)$ & $32(5.8 \%)$ \\
\hline CABG & $4(1.1 \%)$ & $5(0.9 \%)$ \\
\hline $\begin{array}{l}\text { Revascularization after index visit } \\
\text { but within } 30 \text { days }\end{array}$ & $3(0.9 \%)$ & $4(0.7 \%)$ \\
\hline $\mathrm{PCl}$ & $2(0.6 \%)$ & $2(0.4 \%)$ \\
\hline CABG & $1(0.3 \%)$ & $2(0.4 \%)$ \\
\hline 30-Day death & $1(0.3 \%)$ & $3(0.5 \%)$ \\
\hline 30-Day cardiac death & $0(0.0 \%)$ & $1(0.2 \%)$ \\
\hline \multicolumn{3}{|c|}{$\begin{array}{l}\mathrm{CABG}=\text { coronary artery bypass graft; } \mathrm{CAD}=\text { coronary artery disease; } \mathrm{ED}=\text { emergency } \\
\text { department; } \mathrm{IQR}=\text { interquartile range; } \mathrm{MACE}=\text { major adverse cardiac event; } \mathrm{MI}=\text { myocardial } \\
\text { infarction; } \mathrm{PCI}=\text { percutaneous coronary intervention. }\end{array}$} \\
\hline
\end{tabular}

diagnostic accuracy point estimates for the 2-hour algorithm were consistently better than those of the 1-hour algorithm. A similar pattern was recently observed in a recent large comparative analysis. ${ }^{18} \mathrm{We}$ believe that the superior point estimates for the 2-hour algorithm are observed because the 2-hour algorithm uses larger serial change (delta) values, making it less vulnerable to misclassification owing simply to analytic variability. The lack of a statistically significant difference in the sensitivity of the 2-hour algorithm compared with that of the 1-hour algorithm observed in this study is likely a function of an overall small sample size.

Analytic variability arises primarily from two sources: pre-analytical variation (relating to issues occurring prior to a sample analysis that can affect results, including test-ordering, patient preparation, specimen collection, processing, and storage) and analytical variation (relating to inherent inaccuracies of the assay itself). ${ }^{26}$ Suppose a real-world analytical variability of $+/-2 \mathrm{ng} / \mathrm{L}$ per sample. This is much less likely to result in misclassification using the 2-hour algorithm rule-out delta of $<4 \mathrm{ng} / \mathrm{L}$ and a rule-in delta of $\geq 10 \mathrm{ng} / \mathrm{L}$ than the 1-hour algorithm rule-out delta of $<3 \mathrm{ng} / \mathrm{L}$ and a rule-in delta of $\geq 5 \mathrm{ng} / \mathrm{L}$ (see Figure 1). In fact, to improve rule-in specificity, other authors have proposed even much larger rule-in delta cutoffs using the same hs-cTnT assay ( $\geq 16 \mathrm{ng} / \mathrm{L}$ on repeat samples within a 24-hour period). ${ }^{27}$

\section{Strengths and limitations}

Strengths of this study include prospective data collection, a relevant patient population (ED patients requiring $\mathrm{MI}$ rule-out with serial troponin testing in the opinion of an emergency physician), care provider blinding to $1-$ and 2-hour hs-cTnT sample results, comprehensive follow-up, two-physician outcome adjudication, and conduct in realworld clinical and laboratory settings.

The primary limitation of this study is the small sample size, which limits the precision of the estimates that can be generated from it. We were unable to achieve our desired sample size owing largely to local practice patterns that included discharging almost half of patients after a single hs-cTnT assay. Furthermore, logistical issues, including an ethics requirement for physician assessment prior to collecting research samples, made it challenging to collect appropriately timed samples, particularly for 1-hour samples, with 200 fewer being collected. This may have led to selection bias, as patients with higher risk presentations may have been assessed more urgently by a physician and thus more likely to be enrolled in the study. However, the practice of assessing higher risk patients more quickly (making them more likely to be successfully enrolled) would result in higher risk patients being concentrated in the study cohorts. The fact that these criteria performed well despite this adds strength to our results. 


\begin{tabular}{|c|c|c|c|c|c|c|c|c|c|}
\hline Cohort & $\begin{array}{l}\text { Eligible } \\
\text { N (\%) }\end{array}$ & Outcome & $\mathrm{TP}$ & $\mathrm{FP}$ & FN & TN & $\begin{array}{l}\text { Sensitivity } \\
(95 \% \mathrm{Cl})\end{array}$ & $\begin{array}{c}\text { NPV } \\
(95 \% \mathrm{Cl})\end{array}$ & $\begin{array}{c}\text { LR- } \\
(95 \% \mathrm{Cl})\end{array}$ \\
\hline 1-Hour: & $219(62.6 \%)$ & Index MI & 36 & 95 & 1 & 218 & $97.3(85.8,99.9)$ & $99.5(97.5,100)$ & $0.04(0.0,0.3)$ \\
\hline Oh hs-cTnT $<12 \mathrm{ng} / \mathrm{L}$ & & 30D MACE & 38 & 93 & 9 & 210 & $80.9(66.7,90.9)$ & $95.9(92.3,98.1)$ & $0.3(0.2,0.5)$ \\
\hline AND & & 30D MI & 36 & 95 & 1 & 218 & $97.3(85.8,99.9)$ & $99.5(97.5,100)$ & $0.04(0.0,0.3)$ \\
\hline \multirow[t]{2}{*}{$\Delta 0-1 \mathrm{~h}<3 \mathrm{ng} / \mathrm{L}$} & & $30 D$ revascularization & 21 & 110 & 8 & 211 & $72.4(52.8,87.3)$ & $96.3(92.9,98.4)$ & $0.4(0.2,0.8)$ \\
\hline & & 30D cardiac death & 0 & 131 & 0 & 219 & NA & $100(98.3,100)$ & NA \\
\hline 2-Hour: & $351(63.8 \%)$ & Index MI & 63 & 136 & 0 & 351 & $100(91.6,100)$ & $100(98.4,100)$ & $0.00(0.0, N A)$ \\
\hline $0 \mathrm{~h} / 2 \mathrm{~h}$ hs-cTnT $<14 \mathrm{ng} / \mathrm{L}$ & & 30D MACE & 65 & 134 & 13 & 338 & $83.3(73.2,90.8)$ & $96.3(93.8,98.0)$ & $0.23(0.14,0.38)$ \\
\hline AND & & 30D Ml & 63 & 136 & 1 & 350 & $98.4(91.6,100)$ & $99.7(98.4,100)$ & $0.02(0.0,0.2)$ \\
\hline \multirow[t]{2}{*}{$\Delta 0-2 \mathrm{~h}<4 \mathrm{ng} / \mathrm{L}$} & & $30 D$ revascularization & 29 & 170 & 12 & 339 & $70.7(54.5,83.9)$ & $96.6(94.1,98.2)$ & $0.4(0.3,0.7)$ \\
\hline & & 30D cardiac death & 0 & 199 & 1 & 350 & $0(0,97.5)$ & $99.7(98.4,100)$ & $1.6(1.5,1.7)$ \\
\hline
\end{tabular}

The prevalence of index MI $(\sim 11 \%)$ in these cohorts is lower than the original derivation and validation studies, which ranged between $16 \%$ and $17 \%,{ }^{5,7,8}$ likely owing to the exclusion of patients with acute ischemic ECG changes. Sensitivity analysis reveals that if all 168 patients with acute ischemic ECG changes had been included in the 2-hour cohort and diagnosed with index MI, the prevalence of index MI could have been as high as $32.2 \%$. However, because these patients clearly represent a high-risk subgroup, clinical practice would dictate that, even in the presence of normal serial hs-cTnT concentrations, most are likely to be admitted for further evaluation. Our focus on patients without ischemic ECG changes allows an evaluation of algorithm performance in those patients who specifically need troponin testing to diagnose or rule out MI.
Finally, patients with potential alternative presentations of cardiac ischemia (e.g., dyspnea, weakness, back pain, nausea, and abdominal pain) were not included, and it is possible that this systematically underrepresents women, patients with diabetes, elderly patients, and other subgroups who are less likely to report chest pain. However, requiring a chief symptom of chest pain as one of the primary enrolment criteria has been commonplace in the MI diagnostic literature and may prevent dilution of disease prevalence in the cohort when presentations unlikely to be cardiac are included.

\section{Clinical implications}

Based on these data and prior literature, both 1- and 2-hour hs-cTnT algorithms are highly accurate for ruling-in and ruling-out $\mathrm{MI}$ in patients with suspected

\begin{tabular}{|c|c|c|c|c|c|c|c|c|c|}
\hline & $\begin{array}{l}\text { Eligible } \\
\mathrm{N}(\%)\end{array}$ & Outcome & $\mathrm{TP}$ & $\mathrm{FP}$ & $\mathrm{FN}$ & TN & $\begin{array}{l}\text { Specificity } \\
(95 \% \text { Cl) }\end{array}$ & $\begin{array}{c}\text { PPV } \\
(95 \% \mathrm{Cl})\end{array}$ & $\begin{array}{c}\text { LR+ } \\
(95 \% \mathrm{Cl})\end{array}$ \\
\hline 1-Hour: & $37(10.6 \%)$ & Index Ml & 26 & 11 & 11 & 302 & $96.5(93.8,98.2)$ & $70.3(53.0,84.1)$ & $20.0(10.8,37.1)$ \\
\hline Oh hs-cTnT $\geq 52 \mathrm{ng} / \mathrm{L}$ & & 30D MACE & 27 & 10 & 20 & 293 & $96.7(94.0,98.4)$ & $73.0(55.9,86.2)$ & $17.4(9.0,33.6)$ \\
\hline OR & & 30D MI & 26 & 11 & 11 & 302 & $96.5(93.8,98.2)$ & $70.3(53.0,84.1)$ & $20.0(10.8,37.1)$ \\
\hline \multirow[t]{2}{*}{$\Delta 0-1 \mathrm{~h} \geq 5 \mathrm{ng} / \mathrm{L}$} & & 30D revascularization & 14 & 23 & 15 & 298 & $92.8(89.4,95.4)$ & $37.8(22.5,55.2)$ & $6.7(3.9,11.6)$ \\
\hline & & 30D cardiac death & 0 & 37 & 0 & 313 & $89.4(85.7,92.5)$ & $0(0.0,9.5)$ & NA \\
\hline 2-Hour: & $58(10.5 \%)$ & Index MI & 48 & 10 & 15 & 477 & $97.9(96.3,100)$ & $82.8(70.6,91.4)$ & $37.1(19.8,69.6)$ \\
\hline $0 \mathrm{~h} / 2 \mathrm{~h}$ hs-cTnT $\geq 53 \mathrm{ng} / \mathrm{L}$ & & 30D MACE & 48 & 10 & 30 & 462 & $97.9(96.1,99.0)$ & $82.8(70.6,91.4)$ & $29.0(15.3,55.0)$ \\
\hline OR & & 30D MI & 48 & 10 & 16 & 476 & $97.9(96.3,99.0)$ & $82.8(70.6,91.4)$ & $36.5(19.4,68.4)$ \\
\hline \multirow[t]{2}{*}{$\Delta 0-2 \mathrm{~h} \geq 10 \mathrm{ng} / \mathrm{L}$} & & 30D revascularization & 17 & 41 & 24 & 468 & $91.9(89.2,94.2)$ & $29.3(18.1,42.7)$ & $5.2(3.2,8.2)$ \\
\hline & & 30D cardiac death & 0 & 58 & 1 & 491 & $89.4(86.6,91.9)$ & $0(0.0,6.2)$ & $0.0(0.0, N A)$ \\
\hline
\end{tabular}


ischemic chest pain. We strongly encourage the adoption of similar hs-cTn algorithms in EDs across Canada given their proven performance and ability to expedite care and improve the objectivity of evaluation. We believe that 2-hour algorithms offer several practical advantages, given potential challenges collecting appropriately timed 1-hour samples in busy EDs, and because the larger serial change (delta) cutoffs are less susceptible to misclassification secondary to analytic variability. Therefore, we believe that it is most prudent for centres implementing new hs-cTn assays to consider 2-hour algorithms, which, while still facilitating rapid decisionmaking, may be more practical to implement and offer a greater margin of safety - an opinion that is shared by other authors. ${ }^{28,29}$

\section{Research implications}

Despite the excellent performance of the low- and highrisk criteria for both algorithms, approximately onequarter of patients remain in a non-diagnostic "observational zone" after serial troponin testing. Because this cohort has an $\sim 11 \%$ MI prevalence, careful clinical assessment is required to ensure a safe disposition. To date, there are no clear guidelines on how best to manage these patients; however, recommendations for additional serial hs-cTnT sampling to assess for ongoing myocardial injury, careful consideration of alternative diagnoses, and cautious disposition using a validated risk prediction tool, such as the HEART score, would seem prudent. ${ }^{29}$ Validating an evidence-based pathway to safely disposition observational zone patients should be a priority for future research.

\section{CONCLUSIONS}

Both the ESC 1-hour algorithm and an alternative 2-hour hs-cTn T diagnostic algorithm can rapidly and accurately rule-in or rule-out MI for about threequarters of ED patients with chest pain. While no statistically significant differences in diagnostic performance were found between the algorithms, we note the 2-hour algorithm to offer several practical advantages and may be easier to implement in everyday practice. Implementation of hs-cTn algorithms has the potential to significantly decrease ED length of stay and resource utilization. Future research should focus on further comparative analysis of rapid diagnostic algorithms in real- world practice and providing more objective guidance for the observational zone population with indeterminate hs-cTn results.

Supplementary material: The supplemental material for this article can be found at https://doi.org/10.1017/cem.2020.349.

Acknowledgements: We would like to acknowledge the assistance of our research team, including Heidi Boyda, Katrina Koger, and Tiffany Junghans in the completion of this study.

Competing interests: None declared.

Financial support: This research was funded by an investigatorinitiated, unrestricted research grant from Roche Diagnostics Canada. None of the study investigators received any direct or indirect compensation for the conduct of this study.

\section{REFERENCES}

1. Reichlin T, Hochholzer W, Bassetti S, et al. Early diagnosis of myocardial infarction with sensitive cardiac troponin assays. N Engl f Med 2009;361(9):858-67. doi:10.1056/ NEJMoa0900428.

2. Pickering JW, Than MP, Cullen L, et al. Rapid rule-out of acute myocardial infarction with a single high-sensitivity cardiac troponin $\mathrm{T}$ measurement below the limit of detection: a collaborative meta-analysis. Ann Intern Med 2017;166 (10):715-24. doi:10.7326/M16-2562.

3. Andruchow JE, Boyne T, Innes G, et al. Low high-sensitivity troponin thresholds identify low-risk patients with chest pain unlikely to benefit from further risk stratification. CFC Open 2019;1(6):289-96, doi:10.1016/j.cjco.2019.08.002.

4. Roffi M, Patrono C, Collet J-P, et al. 2015 ESC guidelines for the management of acute coronary syndromes in patients presenting without persistent ST-segment elevation: task force for the management of acute coronary syndromes in patients presenting without persistent ST-segment elevation of the European Society of Cardiology (ESC). Eur Heart 7 2016;37(3):267-315. doi:10.1093/eurheartj/ehv320.

5. Reichlin T, Schindler C, Drexler B, et al. One-hour rule-out and rule-in of acute myocardial infarction using highsensitivity cardiac troponin T. Arch Intern Med 2012;172 (16):1211-8. doi:10.1001/archinternmed.2012.3698

6. Cullen L, Mueller C, Parsonage WA, et al. Validation of high-sensitivity troponin $\mathrm{I}$ in a 2-hour diagnostic strategy to assess 30-day outcomes in emergency department patients with possible acute coronary syndrome. $7 \mathrm{Am}$ Coll Cardiol 2013;62(14):1242-9. doi:10.1016/j.jacc.2013.02.078.

7. Reichlin T, Twerenbold R, Wildi K, et al. Prospective validation of a 1-hour algorithm to rule-out and rule-in acute myocardial infarction using a high-sensitivity cardiac troponin T assay. CMA7 2015;187(8):E243-52. doi:10.1503/ cmaj.141349.

8. Reichlin T, Cullen L, Parsonage WA, et al. Two-hour algorithm for triage toward rule-out and rule-in of acute myocardial infarction using high-sensitivity cardiac troponin T. Am 7 Med 2015;128(4):369-79.e4. doi:10.1016/j.amjmed.2014.10.032. 
9. Rubini Gimenez M, Twerenbold R, Jaeger C, et al. Onehour rule-in and rule-out of acute myocardial infarction using high-sensitivity cardiac troponin I. Am $7 \mathrm{Med}$ 2015;128(8):861-70.e4. doi:10.1016/j.amjmed.2015.01.046.

10. Boeddinghaus J, Reichlin T, Cullen L, et al. Two-hour algorithm for triage toward rule-out and rule-in of acute myocardial infarction by use of high-sensitivity cardiac troponin I. Clin Chem 2016;62(3):494-504. doi:10.1373/clinchem.2015. 249508.

11. Jaeger C, Wildi K, Twerenbold R, et al. One-hour rule-in and rule-out of acute myocardial infarction using highsensitivity cardiac troponin I. Am Heart 7 2016;171(1):92102.e1-5. doi:10.1016/j.ahj.2015.07.022.

12. Mokhtari A, Borna C, Gilje P, et al. A 1-h combination algorithm allows fast rule-out and rule-in of major adverse cardiac events. 7 Am Coll Cardiol 2016;67(13):1531-40. doi:10.1016/ j.jacc.2016.01.059.

13. Mueller C, Giannitsis E, Christ M, et al. Multicenter evaluation of a 0-hour/1-hour algorithm in the diagnosis of myocardial infarction with high-sensitivity cardiac troponin T. Ann Emerg Med 2016;68(1):76-87.e4. doi:10.1016/j.annemergmed.2015. 11.013 .

14. Boeddinghaus J, Nestelberger T, Twerenbold R, et al. Direct comparison of 4 very early rule-out strategies for acute myocardial infarction using high-sensitivity cardiac troponin I. Circulation 2017;135(17):1597-611. doi:10.1161/CIRCULATIONAHA. 116.025661 .

15. Lindahl B, Jernberg T, Badertscher P, et al. An algorithm for rule-in and rule-out of acute myocardial infarction using a novel troponin I assay. Heart Br Card Soc 2017;103(2):12531. doi:10.1136/heartjnl-2016-309951.

16. McRae AD, Innes G, Graham M, et al. Comparative evaluation of 2-hour rapid diagnostic algorithms for acute myocardial infarction using high-sensitivity cardiac troponin T. Can 7 Cardiol 2017; 33(8): 1006-1012. doi:10.1016/j.cjca.2017. 04.010 .

17. Wildi K, Cullen L, Twerenbold R, et al. Direct comparison of 2 rule-out strategies for acute myocardial infarction: 2-h accelerated diagnostic protocol vs $2-\mathrm{h}$ algorithm. Clin Chem 2017;63(7):1227-36. doi:10.1373/clinchem.2016.268359.

18. Wildi K, Boeddinghaus J, Nestelberger T, et al. Comparison of fourteen rule-out strategies for acute myocardial infarction. Int 7 Cardiol 2019;283:41-7. doi:10.1016/j.ijcard.2018.11.140.

19. Neumann JT, Twerenbold R, Ojeda F, et al. Application of high-sensitivity troponin in suspected myocardial infarction.
$N$ Engl 7 Med 2019;380(26):2529-40. doi:10.1056/ NEJMoa1803377.

20. Body R, Carlton E. Understanding cardiac troponin part 1: avoiding troponinitis. Emerg Med 7 2017; 35(2): 120-125. doi:10.1136/emermed-2017-206812.

21. Grafstein E, Bullard MJ, Warren D, Unger B; CTAS National Working Group. Revision of the Canadian Emergency Department Information System (CEDIS) presenting complaint list version 1.1. CFEM 2008;10(2):151-73.

22. Ghali WA, Knudtson ML. Overview of the Alberta provincial project for outcome assessment in coronary heart disease. On behalf of the APPROACH investigators. Can 7 Cardiol 2000;16(10):1225-30.

23. Thygesen K, Alpert JS, Jaffe AS, et al. Third universal definition of myocardial infarction. 7 Am Coll Cardiol 2012;60 (16):1581-98. doi:10.1016/j.jacc.2012.08.001.

24. Hicks KA, Tcheng JE, Bozkurt B, et al. 2014 ACC/AHA key data elements and definitions for cardiovascular endpoint events in clinical trials: a report of the American College of Cardiology/American Heart Association Task Force on clinical data standards (writing committee to develop cardiovascular endpoints data standards). Circulation 2015;132 (4):302-61. doi:10.1161/CIR.0000000000000156.

25. Twerenbold R, Jaeger C, Rubini Gimenez M, et al. Impact of high-sensitivity cardiac troponin on use of coronary angiography, cardiac stress testing, and time to discharge in suspected acute myocardial infarction. Eur Heart $\mathcal{F}$ 2016;37 (44):3324-32. doi:10.1093/eurheartj/ehw232.

26. Nichols J. Preanalytical variation. In Clark W (ed. Contemporary practice in clinical chemistry. 2nd ed. Washington, DC: AACC Press; 2010, 1-12.

27. Douville P, Thériault S. Variability of high-sensitivity troponin $\mathrm{T}$ concentrations in emergency settings: impact for the diagnosis of myocardial infarction. Am 7 Clin Pathol 2018;150(1):51-7. doi:10.1093/ajcp/aqy025.

28. Vasile VC, Jaffe AS. High-sensitivity cardiac troponin in the evaluation of possible AMI. American College of Cardiology 2018. Available at: http://\%3a\%2f\%2fwww.acc.org\%2flatestin-cardiology $\% 2$ farticles $\% 2 \mathrm{f} 2018 \% 2 \mathrm{f0} \% 2 \mathrm{f} 16 \% 2 \mathrm{f} 09 \% 2 \mathrm{f} 17$ $\% 2$ fhigh-sensitivity-cardiac-troponin-in-the-evaluation-ofpossible-ami (accessed November 22, 2019).

29. Andruchow JE, Kavsak PA, McRae AD.: Contemporary emergency department management of patients with chest pain: a concise review and guide for the high-sensitivity troponin era. $\operatorname{Can} 7$ Cardiol 2018;34(2):98-108. doi:10.1016/j.cjca.2017.11.012. 